\title{
Investigation on Methods of Determining the Grouting Quality of Embedded Rock Bolts Using High Frequency Guided Waves
}

\author{
Peng Li, Changsuo Zhang ${ }^{(D)}$, and Guanlin Yang \\ College of Mining Engineering, Taiyuan University of Technology, Taiyuan 030024, Shanxi, China \\ Correspondence should be addressed to Changsuo Zhang; zhangchangsuo@tyut.edu.cn
}

Received 19 October 2020; Revised 9 December 2020; Accepted 5 January 2021; Published 21 January 2021

Academic Editor: Zhijie Wen

Copyright (C) 2021 Peng Li et al. This is an open access article distributed under the Creative Commons Attribution License, which permits unrestricted use, distribution, and reproduction in any medium, provided the original work is properly cited.

\begin{abstract}
Two experiments, with differing equipment setups, were used to test rock bolts, with differing structures and grouting qualities, using low frequency $(20-200 \mathrm{kHz})$ and high frequency $(700 \mathrm{kHz}-3 \mathrm{MHz})$ guided waves to determine the effect of grouting quality on the propagating velocity of the guided waves. The results indicate that grouting quality has a significant effect on the velocity at which waves of low frequencies propagate through embedded rock bolts. As guided wave frequency increases, the sensitivity of the propagating velocity of guided waves to grouting quality decreases. Furthermore, the influence of grouting quality on propagating velocity becomes negligible once the frequency of the guided wave is greater than or equal to $1.0 \mathrm{MHz}$. An investigation was conducted to ascertain the feasibility of utilizing high frequency guided waves to determine the grouting quality of embedded rock bolts. Moreover, this study discusses a method of evaluating the grouting quality of embedded rock bolts using the peak ratios and average amplitude ratios of the high frequency guided waves. Through an analysis of the results of the abovementioned method, it was discovered that the optimal guided wave frequency is $2.65 \mathrm{MHz}$ for the evaluation of $20 \mathrm{~mm}$ fully-embedded rock bolts because waves with this frequency have the largest average amplitude ratios.
\end{abstract}

\section{Introduction}

The traditional and most ubiquitous means of providing support in underground engineering is the utilization of embedded rock bolts. The embedded rock bolts are hidden [1-3] from sight because they are always installed inside the rock face; therefore, it is impossible to directly determine the in-situ grouting quality of an embedded rock bolt. The inability to efficiently and accurately determine in-situ grouting quality can limit the applicability of rock bolts. Embedded rock bolts are widely used and often the conditions are unfavorable for the maintenance of high grouting quality. The grouting quality is affected by environmental factors such as groundwater with various chemical compositions and nearby mining activity; moreover, the groundwater can rust and corrode the rock bolt and nearby mining activity could easily destroy already corroded rock bolts and even fracture noncorroded rock bolts. In addition, some construction companies purposefully cut corners by using rock bolts that cannot meet the required standards to illegally obtain additional profit. This can hasten the eventual failure of the rock bolts due to the abovementioned weakening phenomena and cause potential engineering hazards and disasters. Changes in grouting quality and bolt integrity due to the abovementioned phenomena are hard to detect using conventional methods; therefore, there is a dire need for a new practical and nondestructive test to be designed.

Currently, the commonly used nondestructive test for rock bolts [4-6] involves sending guided waves into the free end of the embedded rock bolt, the guided waves propagate through the rock bolt, the guided waves are reflected off the bolt end, and finally the "echoes" are received by a sensor at the free end of the embedded rock bolt. The propagation velocity of the wave is used to calculate the length of the bolt and the attenuation of the wave is used to determine the grouting quality of the embedded rock bolt [7-9]. This approach can get the job done, but it is not perfect due to the complexity of the wave's propagation in the bolt [10]. The propagation of the wave in the embedded rock bolt is affected by the properties of the bolt, anchoring medium, and 
surrounding rock. Guided waves with differing modes and frequencies exhibit different propagation velocities and degrees of attenuation [11-13]. The echoes of waves which are sensitive to the physical and mechanical properties at the steel-grout interface cannot be received because most of the energy dissipates in the anchoring medium and the surrounding rock [14]. Therefore, conventional nondestructive tests do not have a wide range of applicability and are not widely adopted. This paper discusses a novel method of testing the grouting quality of an embedded rock bolt using high frequency and low attenuation guided waves $(1.5 \mathrm{MHz}$ or higher) [15-17].

\section{Methods}

In this experiment, the guided wave frequency ranges from $20 \mathrm{kHz}$ to $3 \mathrm{MHz}$, and two experimental systems, one for low frequencies $(20 \mathrm{kHz}-200 \mathrm{kHz})$ and one for high frequencies $(0.7 \mathrm{MHz}-3 \mathrm{MHz})$ were used to complete the test. The low frequency experiment uses the equipment setup shown in Figure 1 . The desired waveform generated from the arbitrary waveform generator is input into the transmitter that is fixed at the free end of the rock bolt, then the waveform travels through the rock bolt and is received with a sensor that is fixed at the other side of the rock bolt. The sensors used for these tests are acoustic emission sensors with high sensitivity. The characteristic of this system is that the frequency and waveform of the input wave can be altered according to the rock bolt structure, and the input and output do not affect each other. The drawback of this system is that the experimental conditions deviate from the practical conditions or field conditions in which the rock bolts are used; nevertheless, it can still adequately study the propagation characteristics of guided waves in a rock bolt. A computer program is used to control the pulse transmitter receiver (ultrasonic card) and record the waveform. The wave emitted by an ultrasonic card is a square wave, and the number of waves and frequencies of the waves can be controlled by program [18]. The system has the advantages of being able to use high voltages $(300 \mathrm{~V})$ to send waves with a signal bandwidth of $0.7 \mathrm{MHz}-12 \mathrm{MHz}$, which is suitable for testing with a variety of frequency sensors, and the single sensor system for the high frequency experiment meets the basic requirements of field rock bolt test. The disadvantage of the system is that it can only produce rectangular or square waves, and the output signal becomes distorted when the number of periods is small. The wave frequency range generated by the sensor is broad, but the width of the range makes signal recognition difficult. It can be seen from the dispersion equation of the free [19] end of the rock bolt that the propagation velocity and attenuation characteristics of guided waves in a rock bolt are related to the product of frequency of the guided wave and the diameter of anchor rod [5] (the range of the rock bolt diameter used in the field is $16-24 \mathrm{~mm}$ ). Therefore, the broad range of frequencies enables the testing of rock bolts with a broad range of diameters.

The material used as the anchoring medium for this experiment is cement. \#425 cement and coarse sand were chosen for the anchoring medium according to the strength requirement for anchoring mediums. The ratio of water to cement to sand is $0.5: 1: 1.5$. The diameter of the PVC tube used in the experiment is $20 \mathrm{~cm}$; the diameter of rock bolts in all specimens is $20 \mathrm{~mm}$. Specimen \#1 is a free rock bolt with a length of $68 \mathrm{~cm}$. Specimen \#2 is an embedded rock bolt with a length of $1 \mathrm{~m}$ with a $30 \mathrm{~cm}$ sand layer in the middle. Specimen \#3 is an embedded rock bolt with a length of $70 \mathrm{~cm}$. Specimen \#4 is an embedded rock bolt with a length of $1 \mathrm{~m}$. Specimen \#5 is a semiembedded rock bolt with a length of $1.5 \mathrm{~m}$, the length of the embedded section is $70 \mathrm{~cm}$, and the length of free section is $80 \mathrm{~cm}$. All specimens are shown in Figure 2.

\section{Results and Discussion}

3.1. Relationship between the Guided Wave Frequency and the Guided Wave Velocity. Using the equipment setup, the fullyembedded rock bolt (specimen \#4) and the free rock bolt (specimen \#1) were scanned at low frequency $(20 \sim 200 \mathrm{kHz})$, and the results of the experiment were plotted in Figure 3.

The difference in propagation velocity between the fullyembedded rock bolt and the free rock bolt is quite large, and the basic trend, in Figure 3, is that the velocity difference decreases as the frequency increases. Thus, it can be inferred that the propagation velocity of low frequency guided waves is sensitive to the grouting quality, and the influence of the grouting quality on the propagation velocity of guided waves decreases as the frequency increases. Therefore, the grouting quality can be measured using low frequency guided waves by calculating the difference between the two velocities.

Using the experiment setup, the fully-embedded rock bolt (specimen \#4) and the free rock bolt (specimen \#1) were scanned at high frequency $(0.7 \sim 3 \mathrm{MHz})$, and the results of the experiment are plotted in Figure 4. Furthermore, scans using frequencies in the range of $200-700 \mathrm{kHz}$ were not performed due to equipment limitations.

The difference between the guided wave velocity in the free rock bolt and the guided wave velocity in the embedded rock bolt decreases further as the guided wave frequency increases. The two velocities begin to quickly converge in the frequency range of $1-3 \mathrm{MHz}$, and the two velocities converge at frequencies that are slightly higher than $1 \mathrm{MHz}$. This shows that the propagation velocity of guided waves is not significantly affected by the characteristics of the anchoring medium in the frequency range of $1 \sim 3 \mathrm{MHz}$. The attenuation of low frequency guided waves in the experiment specimens is large and echoes are hard to detect because the specimens are too long and allow the waves to disperse before detection, therefore guided waves with frequencies in the range of $1-3 \mathrm{MHz}$ are most suitable for determining the integrity of rock bolts. This paper mainly focuses on discussing why the high frequency guided wave is used to detect the anchoring quality of anchor rod.

3.2. Velocity Analysis of Ultrasonic Guided Waves with Different Anchorage Quality. The results in Figure 4 show that the wave velocities in free rock bolts and embedded rock bolts 


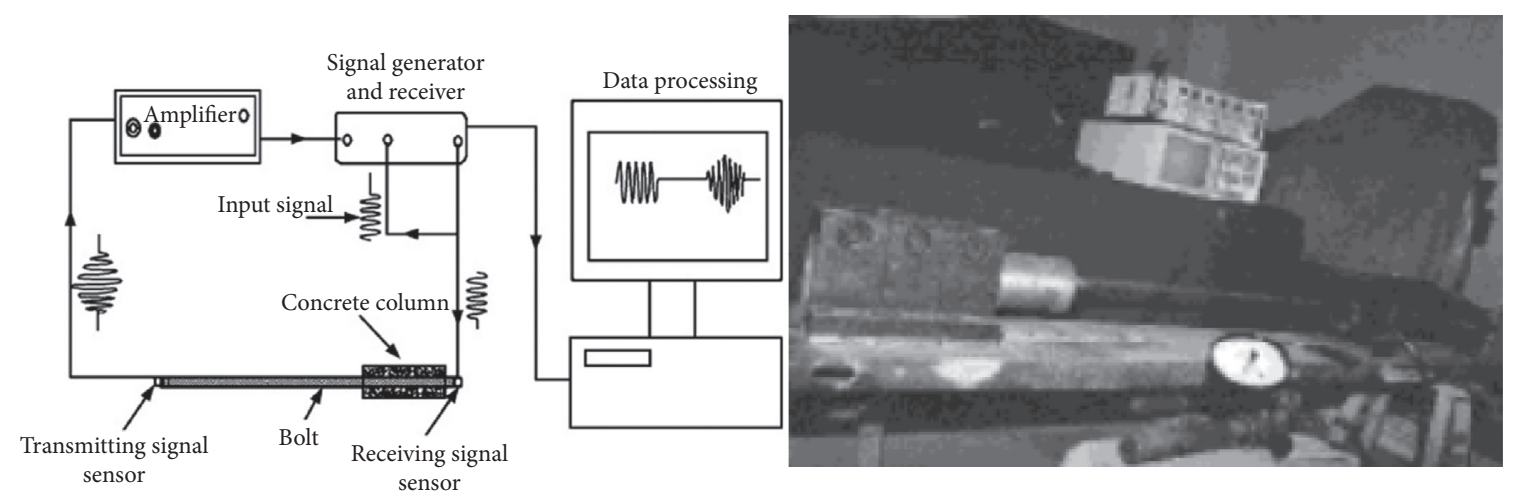

FIGURE 1: Variable frequency wave creator with single sensor system diagram.
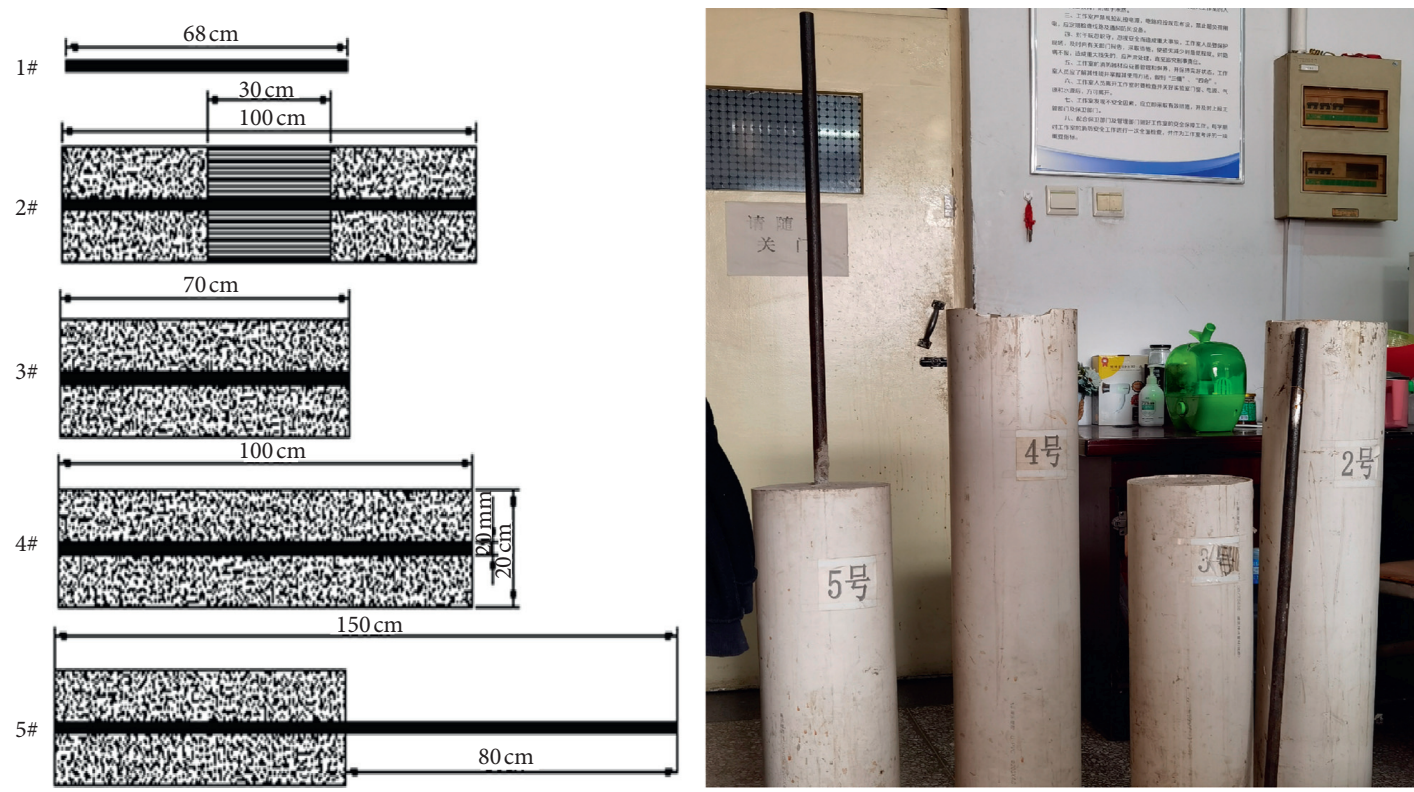

Figure 2: Diagram of specimens 1-5.

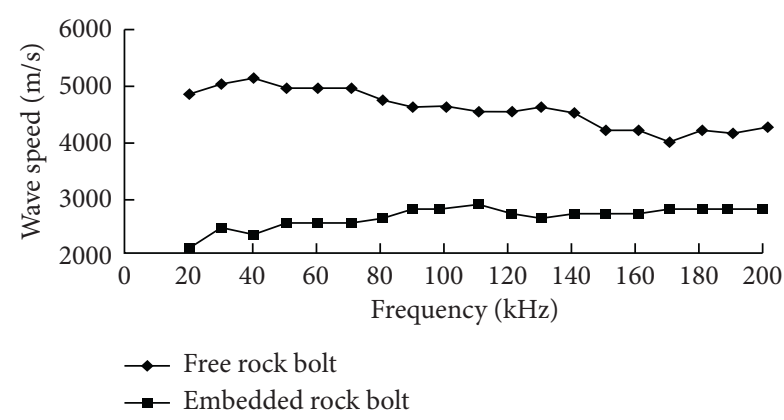

FIGURE 3: Low frequency guided wave propagation velocity in free and embedded rock bolt.

are the same, however one must further analyze this phenomena to determine its legitimacy. In order to further study the effect of the progressive change of the grouting quality on the propagation velocity of high frequency guided waves, specimen \#4 was progressively tested, at different times in the curing process [20], using high frequency waves after the anchoring medium was poured. During the 28-day curing

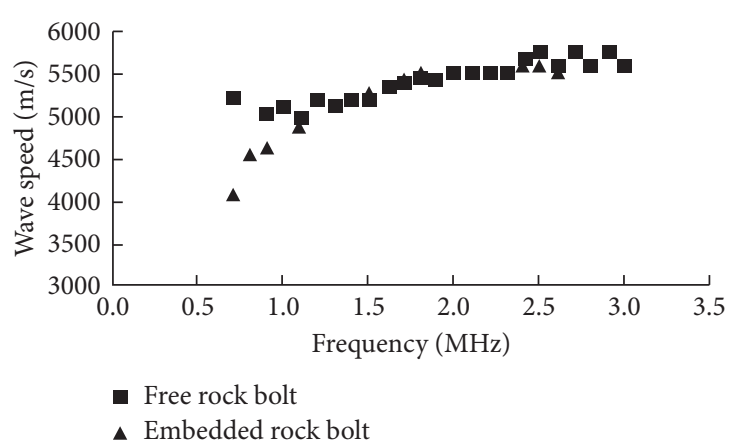

FIGURE 4: High frequency guided wave propagation velocity in free and embedded rock bolt.

period, tests were conducted every six hours throughout the first seven days, and tests were conducted every 12 hours throughout the subsequent three days, thereafter tests were conducted every 24 hours. To determine the frequencies of the echoes, FFT transforms were conducted on different wave packets. The results are shown in Figure 5. 
The results of the FFT transforms indicate that, for wave packets with the same frequencies, there is no significant wavelet packet velocity change as the curing progresses. Furthermore, the results indicate that high frequency guided waves have greater propagation velocities than low frequency guided waves. A guided wave with a frequency of $3.372 \mathrm{MHz}$ yielded the maximum propagation velocity of $5830 \mathrm{~m} / \mathrm{s}$, and a guided wave with a frequency of $1.734 \mathrm{MHz}$ yielded the minimum propagation velocity of $5433 \mathrm{~m} / \mathrm{s}$. The experimental results for specimen \# 5 corroborate the results from specimens \#1 and \#4. Therefore, this reiterates the fact that the utilization of velocity difference of high frequency guided waves is not a feasible method of determining grouting quality. The changes in grouting quality are reflected in the changes in the tightness between the anchoring medium, surrounding rock, and anchor rod. Therefore, more energy is transferred from the rock bolt to the anchorage medium and surrounding rock if the grouting quality is high. Moreover, it is possible to use the attenuation of guided waves to determine the grouting quality of rock bolts.

\subsection{Ultrasonic Guided Wave Attenuation Analysis Method.}

Two methods are used to measure the attenuation of a rock bolt. One is the peak ratio method, which is the ratio of the second reflected wave packet peak to the first reflected wave packet peak [21]. The other is the average amplitude ratio method, which is the ratio of the average amplitude of the second reflected wave packet to the average amplitude of the first reflected wave packet.

3.3.1. Peak Ratio Method. The peak values of the first and second reflected wave packets are shown in Figure 6. If the peak value of the first reflected wave packet is A1 and the second reflected wave packet's peak value is A2, then the peak ratio is

$$
R=\frac{A_{2}}{A_{1}} .
$$

Waveforms that utilize varying gains were recorded in the experiment. When the recorded waveform has two reflected wave packets that utilize the same gain, the peak value of the second reflected wave packet is directly divided by the peak value of the first reflected wave packet to determine the peak ratio. On the other hand, the peak ratio cannot be directly calculated if the first wave packet and the second wave packet utilize two different gains. The true voltage value can be obtained by dividing the amplified peak value of the second reflected wave packet by the magnification factor; furthermore, the peak ratio can be determined by dividing the true value of the second reflected wave packet by the true value of the second reflected wave packet. The peak values and peak ratios of specimens $1 \#-5 \#$ are tabulated in Table 1 and the peak ratios are plotted in Figure 7.

It can be seen from Figure 7 that the peak ratio is different due to the difference in the grouting quality of the specimen, and the peak ratio does not change as gain increases. The peak

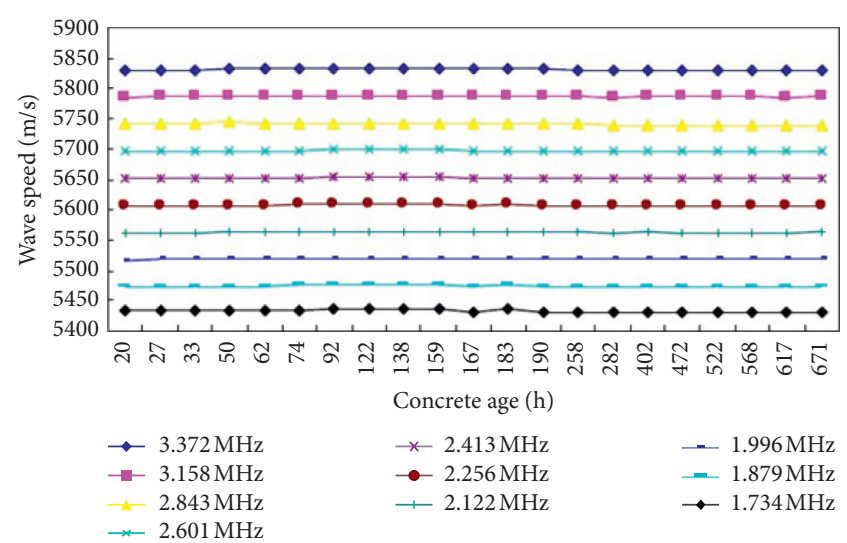

FIgURE 5: Guided wave speed of \#4 specimen with different frequency guided waves at different times in the curing process.

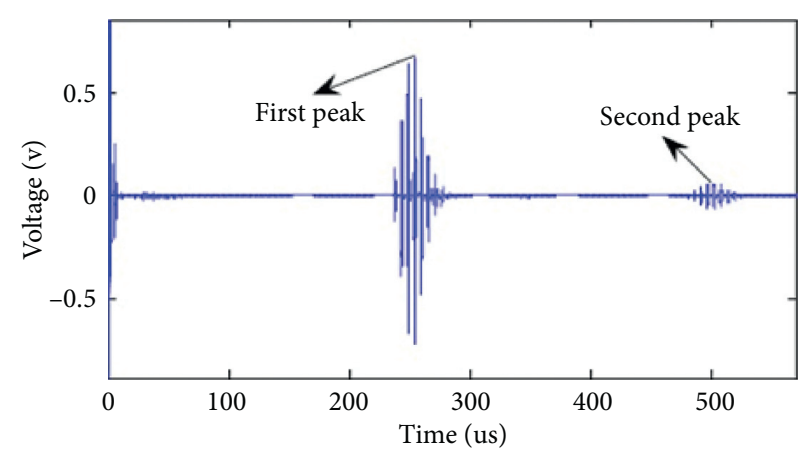

Figure 6: Peak ratio method.

ratio of specimen \#1 is $0.16-0.18$, which is the largest peak ratio value among the five specimens. Specimen \#1 is a free rock bolt, which is not affected by the surrounding cement mortar anchoring medium. Its grouting quality can be regarded as the worst among the five specimens. Specimens $\# 2$ and \#4 can be compared because the rods are of the same length. Specimen \#2 contains a sand layer, which causes the grouting quality to be significantly lower than the fully grouted specimen \#4, therefore specimen \#2's peak ratio is greater than that of specimen \#4. Specimens \#1 and \#3 are representatives of specimens with the worst grouting quality and the best grouting quality, respectively. It can be seen inferred from the analyses of specimens \#1, \#2, and \#4 that the peak ratio decreases as the grouting quality increases. The peak ratio of the \#5 test piece is the smallest, which is caused by the combination of its length and being semigrouted. Specimens \#3 and \#4 are both fully grouted and specimen \#3's peak ratio is greater than that of specimen \#4 because the rock bolt lengths are different.

3.3.2. Average Amplitude Ratio Method. In this paper, the concept of mean amplitude is used to represent the energy of the reflected wave packet, and the average amplitude ratio method is used to measure the attenuation of guided waves in the free rock bolt and embedded rock bolt. The formula for calculating the average amplitude is as follows: 
TABLe 1: 1\#-5\# peak ratio data.

\begin{tabular}{|c|c|c|c|c|}
\hline Specimen & Gain $(\mathrm{dB})$ & Peak value of the first wave packet & Peak value of the second wave packet & Peak ratio \\
\hline \multirow{5}{*}{$1 \#$} & 20 & 0.651 & 0.109 & 0.167 \\
\hline & 22 & 0.839 & 0.141 & 0.168 \\
\hline & 25 & 1.171 & 0.200 & 0.171 \\
\hline & 30 & 2.083 & 0.354 & 0.170 \\
\hline & 35 & 3.704 & 0.639 & 0.173 \\
\hline \multirow{6}{*}{$2 \#$} & 20 & 0.640 & 0.037 & 0.059 \\
\hline & 25 & 1.139 & 0.073 & 0.064 \\
\hline & 30 & 2.025 & 0.135 & 0.067 \\
\hline & 35 & 3.601 & 0.247 & 0.069 \\
\hline & 40 & 6.404 & 0.4441 & 0.069 \\
\hline & 45 & 11.388 & 0.784 & 0.069 \\
\hline \multirow{7}{*}{$3 \#$} & 5 & 0.634 & 0.049 & 0.078 \\
\hline & 8 & 0.890 & 0.072 & 0.081 \\
\hline & 10 & 1.123 & 0.093 & 0.083 \\
\hline & 15 & 1.998 & 0.170 & 0.085 \\
\hline & 20 & 3.552 & 0.237 & 0.067 \\
\hline & 25 & 6.317 & 0.427 & 0.068 \\
\hline & 30 & 11.233 & 0.754 & 0.067 \\
\hline \multirow{7}{*}{$4 \#$} & 20 & 0.453 & 0.020 & 0.044 \\
\hline & 25 & 0.804 & 0.040 & 0.050 \\
\hline & 30 & 1.431 & 0.075 & 0.053 \\
\hline & 35 & 2.545 & 0.138 & 0.054 \\
\hline & 40 & 4.525 & 0.248 & 0.055 \\
\hline & 45 & 8.047 & 0.440 & 0.055 \\
\hline & 50 & 14.310 & 0.766 & 0.054 \\
\hline \multirow{8}{*}{$5 \#$} & 35 & 0.558 & 0.013 & 0.024 \\
\hline & 40 & 0.979 & 0.028 & 0.029 \\
\hline & 45 & 1.752 & 0.053 & 0.030 \\
\hline & 50 & 3.116 & 0.096 & 0.031 \\
\hline & 55 & 5.541 & 0.179 & 0.032 \\
\hline & 60 & 9.853 & 0.313 & 0.032 \\
\hline & 65 & 17.521 & 0.476 & 0.027 \\
\hline & 70 & 31.158 & 0.747 & 0.024 \\
\hline
\end{tabular}

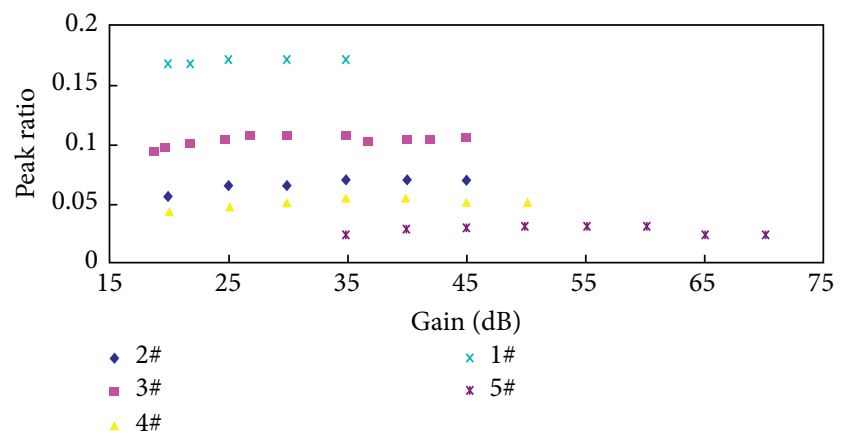

Figure 7: Peak ratios of specimens 1-5\#.

$A_{i}=\sqrt{\frac{\int_{t_{i 1}}^{t_{i 2}} k v_{i}^{2}(t) \mathrm{d} t}{(1 / 2) k\left(t_{i 2}-t_{i 1}\right)}}=\sqrt{\frac{2}{\left(t_{i 2}-t_{i 1}\right)} \int_{t_{i 1}}^{t_{i 2}} v_{i}^{2}(t) \mathrm{d} t} \quad, i=1,2$,

where $\Delta t_{i}=t_{i 2}-t_{i 1}$ is the selected time interval; $v_{i}(t)$ is the record signal strength $(v) ; i=1$ represents the first reflected wave packet; $i=2$ represents the second reflected wave packet; and $K$ is the constant.
The average amplitude ratio of the first and second reflected wave packets is

$$
R_{m}=\frac{A_{2}}{A_{1}}
$$

where A1 is the average amplitude of the first reflected wave packet, and A2 is the average amplitude of the second reflected wave packet.

Figure 8 is a waveform diagram with two reflections in the test waveform. Figure 9 is the magnified waveform of the first reflected wavelet packet; moreover, many wavelet packets can be seen in the graph. Before calculating the average amplitude ratio, FFT transforms were performed on the wavelet packets of both first and second magnified reflected waveforms to determine which wavelet packets have similar frequencies in both reflected waveforms. $\Delta \mathrm{t} 1, \Delta \mathrm{t} 2$ are the time intervals or periods of wavelet packets with similar frequencies in the first reflected wave and the second reflected wave, respectively. In the calculation process, the smaller period of $\Delta \mathrm{t} 1$ and $\Delta \mathrm{t} 2$ is taken as the calculation time for the average amplitude ratio of the two reflected wave packets. Then, the average amplitude of the first reflected wave packet and the second reflected wave packet can be 


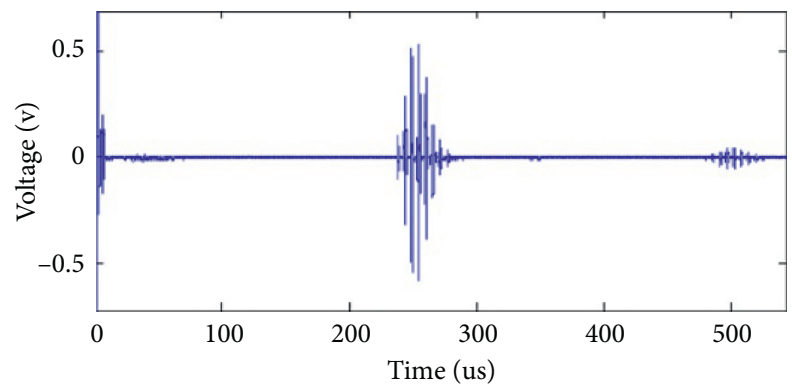

Figure 8: Two reflection waveforms.

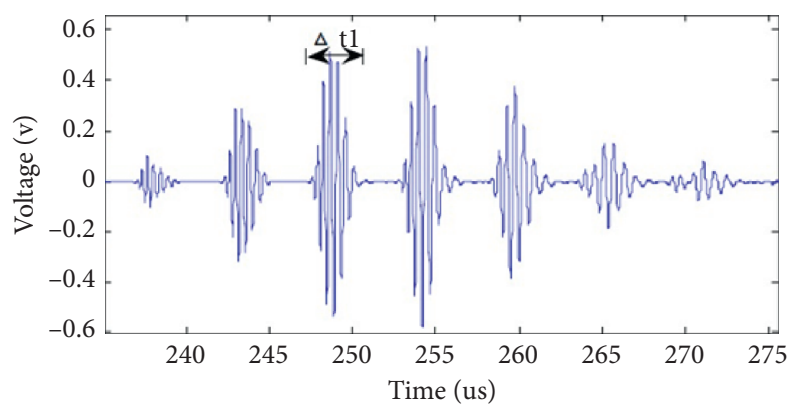

FIgUre 9: Amplified waveform of the first wave packet from Figure 8.

TABLE 2: 1\#-5\# average amplitude ratio data.

\begin{tabular}{|c|c|c|c|c|c|c|c|c|c|}
\hline \multirow{6}{*}{$1 \#$} & Frequency/MHz & Gain/dB & 20 & 22 & 25 & 30 & 35 & & \\
\hline & 2.436 & \multirow{5}{*}{ Average amplitude ratio } & 0.349 & 0.352 & 0.361 & 0.335 & 0.373 & & \\
\hline & 2.118 & & 0.170 & 0.171 & 0.173 & 0.162 & 0.177 & & \\
\hline & 1.889 & & 0.177 & 0.179 & 0.1819 & 0.171 & 0.185 & & \\
\hline & 1.66 & & 0.196 & 0.198 & 0.200 & 0.188 & 0.205 & & \\
\hline & 1.47 & & 0.212 & 0.214 & 0.217 & 0.206 & 0.222 & & \\
\hline \multirow{7}{*}{$2 \#$} & Frequency/MHz & \multirow{7}{*}{ Average amplitude ratio } & 20 & 25 & 30 & 35 & 40 & 45 & \\
\hline & 2.688 & & 0.128 & 0.132 & 0.136 & 0.140 & 0.142 & 0.142 & \\
\hline & 2.532 & & 0.086 & 0.090 & 0.093 & 0.096 & 0.097 & 0.097 & \\
\hline & 2.273 & & 0.082 & 0.087 & 0.090 & 0.090 & 0.092 & 0.092 & \\
\hline & 2.161 & & 0.070 & 0.075 & 0.077 & 0.079 & 0.079 & 0.079 & \\
\hline & 1.938 & & 0.056 & 0.060 & 0.063 & 0.065 & 0.066 & 0.066 & \\
\hline & 1.819 & & 0.060 & 0.065 & 0.068 & 0.071 & 0.072 & 0.072 & \\
\hline \multirow{7}{*}{$3 \#$} & Frequency/MHz & \multirow{7}{*}{ Average amplitude ratio } & 5 & 8 & 10 & 15 & 20 & 25 & 30 \\
\hline & 2.594 & & 0.372 & 0.379 & 0.378 & 0.386 & 0.326 & 0.331 & 0.330 \\
\hline & 2.438 & & 0.147 & 0.151 & 0.151 & 0.153 & 0.126 & 0.127 & 0.127 \\
\hline & 2.302 & & 0.0901 & 0.093 & 0.093 & 0.095 & 0.076 & 0.077 & 0.077 \\
\hline & 2.135 & & 0.070 & 0.073 & 0.073 & 0.075 & 0.059 & 0.060 & 0.060 \\
\hline & 1.884 & & 0.043 & 0.045 & 0.045 & 0.048 & 0.037 & 0.038 & 0.039 \\
\hline & 1.651 & & 0.051 & 0.052 & 0.052 & 0.055 & 0.043 & 0.045 & 0.046 \\
\hline \multirow{8}{*}{$4 \#$} & Frequency/MHz & \multirow{8}{*}{ Average amplitude ratio } & 20 & 25 & 30 & 35 & 40 & 45 & 50 \\
\hline & 2.623 & & 0.111 & 0.107 & 0.110 & 0.112 & 0.112 & 0.112 & 0.110 \\
\hline & 2.425 & & 0.073 & 0.075 & 0.076 & 0.078 & 0.078 & 0.077 & 0.076 \\
\hline & 2.278 & & 0.060 & 0.062 & 0.065 & 0.065 & 0.065 & 0.065 & 0.064 \\
\hline & 2.149 & & 0.050 & 0.053 & 0.055 & 0.056 & 0.056 & 0.056 & 0.055 \\
\hline & 1.98 & & 0.050 & 0.053 & 0.056 & 0.057 & 0.058 & 0.058 & 0.057 \\
\hline & 1.774 & & 0.041 & 0.040 & 0.042 & 0.044 & 0.046 & 0.046 & 0.045 \\
\hline & 1.62 & & 0.056 & 0.048 & 0.048 & 0.050 & 0.051 & 0.052 & 0.052 \\
\hline \multirow{8}{*}{$5 \#$} & Frequency/MHz & \multirow{8}{*}{ Average amplitude ratio } & 40 & 45 & 50 & 55 & 60 & 65 & 70 \\
\hline & 2.745 & & 0.042 & 0.035 & 0.032 & 0.036 & 0.041 & 0.039 & 0.040 \\
\hline & 2.494 & & 0.049 & 0.048 & 0.048 & 0.050 & 0.052 & 0.046 & 0.043 \\
\hline & 2.367 & & 0.038 & 0.038 & 0.038 & 0.039 & 0.040 & 0.034 & 0.032 \\
\hline & 2.245 & & 0.040 & 0.039 & 0.039 & 0.041 & 0.040 & 0.034 & 0.031 \\
\hline & 2.149 & & 0.034 & 0.034 & 0.034 & 0.035 & 0.035 & 0.030 & 0.027 \\
\hline & 2.004 & & 0.033 & 0.034 & 0.034 & 0.035 & 0.035 & 0.030 & 0.027 \\
\hline & 1.874 & & 0.028 & 0.029 & 0.029 & 0.030 & 0.030 & 0.026 & 0.024 \\
\hline
\end{tabular}




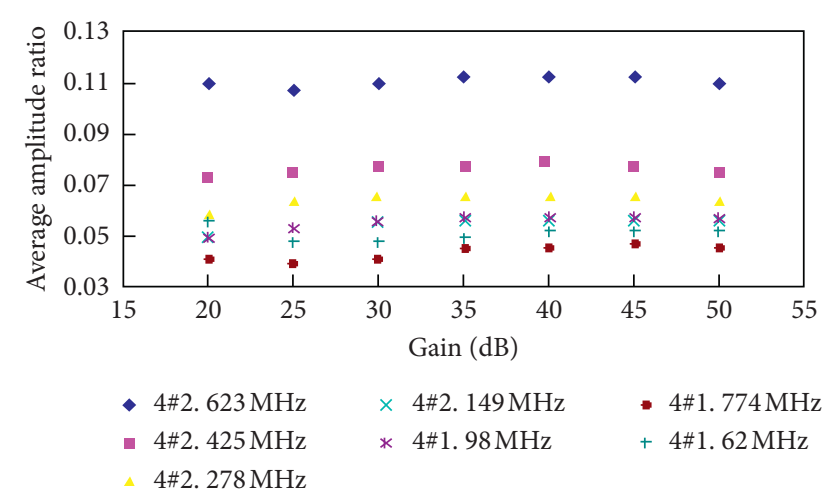

Figure 10: Average amplitude ratio vs gain for specimen \#4 at different frequencies.

calculated, and finally the average amplitude ratio can be calculated. The average amplitude ratios at different gains and different frequencies were recorded for each of the specimens and were tabulated into Table 2. Figure 10 shows the plot of gain vs average amplitude ratio at different frequencies for specimen \#4 using the data tabulated in Table 2.

By comparing the average amplitude ratio and peak ratio of a wave with the optimal frequency, $2.623 \mathrm{MHz}$, in specimen \#4, the former is approximately two times greater than the latter. Therefore, a transmitter that can create a wave with a singular optimal frequency would be able to evaluate a rock bolt with a much longer length.

\section{Conclusions}

To design a new nondestructive method of evaluating the insitu grouting quality of embedded rock bolts, two experiments with different equipment setups were used to test embedded rock bolts with differing grouting qualities and structures using low frequency and high frequency guided waves. The main conclusions were drawn as follows:

(1) At low frequencies $(20 \mathrm{kHz}-1 \mathrm{MHz})$, the sensitivity of the propagating velocity of guided waves to grouting quality is high and the sensitivity decreases as frequency increases. Once the frequency reaches $1 \mathrm{MHz}$ or higher, the influence of grouting quality on the propagating velocity of guided waves is negligible. Therefore, it is impossible to determine grouting quality using the changes in the propagating velocity of high frequency guided waves.

(2) As the grouting quality increases, regardless of frequency of the guided waves, the peak ratio and average amplitude ratios decrease. These two ratios can be used to evaluate the grouting quality of embedded rock bolts.

(3) For rock bolts with a diameter of $20 \mathrm{~mm}$, a guided wave with a frequency of $2.65 \mathrm{MHz}$ has the least attenuation. This frequency allows the guided wave to travel the furthest (i.e., it is able to test the longest rod); therefore, this frequency is the most optimal frequency that can be utilized to test $20 \mathrm{~mm}$ embedded rock bolts. Furthermore, the distance and length of the high-frequency guided wave test will increase when the wave with the singular optimal frequency is used in the test.

\section{Data Availability}

The data used to support the findings of this study are included within the article.

\section{Conflicts of Interest}

The authors declare that there are no conflicts of interest regarding the publication of this paper.

\section{Acknowledgments}

This study was supported by the opening project of State Key Laboratory of Explosion Science and Technology (Beijing Institute of Technology) (Grant no. KFJJ17-09M). The authors would like to thank Ruihao Zhao for his assistance in translating and editing this paper.

\section{References}

[1] S. Lu and Anchorage Force and Anchorage Technology of Bolt, China Coal Industry, Publishing House, Beijing, China, 1998.

[2] T. Liang, Technical Manual for Anchorage and Grouting, China Electric Power Press, Beijing, China, 1999.

[3] C. Hou, L. Guo, and P. Gou, Coal Roadway Bolt Support, China University of Mining and Technology Press, Xuzhou, China, 1999.

[4] M. D. Bread and M. J. S. Lowe, "Non-destructive testing of rock bolts using guided ultrasonic waves," International Journal of Rock Mechanics and Mining Sciences, vol. 40, pp. 78-81, 2003.

[5] M. D. Bread, Guided Wave Inspection of Embedded Cylindrical Structures, Imperial College of Science Technology and Medicine, London, England, 2002.

[6] F. Guo and C. Zhang, "Non-destructive testing technology and research progress of anchorage quality of anchorage rod," Journal of Taiyuan University of Technology, vol. 36, no. 4, pp. 11-13, 2005.

[7] M. Wang and H. Wang, "Nondestructive testing of anchoring quality," Chinese Journal of Rock Mechanics and Engineering, vol. 1, pp. 126-129, 2002.

[8] Li Yi and C. Wang, "Experiment study on bolt bonding integrity with stress reflected wave method," Journal of China Coal Society, vol. 2, pp. 160-164, 2000.

[9] D. H. S. Zou, J. Cheng, R. Yue, and X. Sun, "Grout quality and its impact on guided ultrasonic waves in grouted rock bolts," Journal of Applied Geophysics, vol. 72, no. 2, pp. 102-106, 2010.

[10] C. Wang, W. He, J. Ning, and C. Zhang, "Propagation properties of guided wave in the anchorage structure of rock bolts," Journal of Applied Geophysics, vol. 69, no. 3-4, pp. 131-139, 2009.

[11] Y. Cui and D. H. Zou, "Numerical simulation of attenuation and group velocity of guided ultrasonic wave in grouted rock bolts," Journal of Applied Geophysics, vol. 59, no. 4, pp. 337-344, 2006. 
[12] J. L. Rose, M. J. Avioli Jr, and Y. Cho, "Elastic wave analysis for broken rail detection," American Institute of Physics, vol. 615, pp. 1806-1812, 2002.

[13] P. Wilcox, M. Lowe, and P. Cawley, "An EMAT array for the rapid inspection of large structures using guided waves," Review of Progress in Quantitative Nondestructive Evaluation, vol. 657, no. 1 , pp. 814-821, 2003.

[14] S. Pavlakovic and B. Nicholas, Leaky Guided Ultrasonic Waves in NDT, Imperial College London (University of London), London, England, 1998.

[15] C. He, "Propagation characteristics of high frequency longitudinal ultrasonic guided waves in steel rod embedded in infinite medium," Chinese Journal of Mechanical Engineering, vol. 39, no. 3, pp. 538-544, 2007.

[16] B. Wu, Y. Sun, C. He, X. Wang, Z. Liu, and Q. Zhang, "Application of high frequency ultrasonic guided waves to inspection of full-length-bonding bolt," Chinese Journal of Rock Mechanics \& Engineering, vol. 26, no. 2, pp. 397-403, 2007.

[17] B. N. Pavlakovic, M. J. S. Lowe, and P. Cawley, "High-frequency low-loss ultrasonic modes in imbedded bars," Journal of Applied Mechanics, vol. 68, no. 1, pp. 67-75, 2001.

[18] T. Yamasaki, S. Tamai, and M. Hirao, "Optimum excitation signal for long-range inspection of steel wires by longitudinal waves," NDT \& E International, vol. 34, no. 3, pp. 207-212, 2001.

[19] J. L. Rose and P. B. Nagy, "Ultrasonic waves in solid media," The Journal of the Acoustical Society of America, vol. 107, no. 4, pp. 1807-1808, 2000.

[20] V. Madenga, D. H. Zou, and C. Zhang, "Effects of curing time and frequency on ultrasonic wave velocity in grouted rock bolts," Journal of Applied Geophysics, vol. 59, no. 1, pp. 79-87, 2006.

[21] C. S. Zhang, D. H. Zou, and V. Madenga, "Numerical simulation of wave propagation in grouted rock bolts and the effects of mesh density and wave frequency," International Journal of Rock Mechanics and Mining Sciences, vol. 43, no. 4, pp. 634-639, 2006. 